\title{
Higher order functional boundary value problems without monotone assumptions
}

\author{
João F Fialho ${ }^{1,3^{*}}$ and Feliz Minhós $s^{2,3}$
}

\author{
"Correspondence: \\ jfzero@gmail.com \\ 'School of Mathematics, Physics \\ and Technology, College of the \\ Bahamas, Nassau, Bahamas \\ ${ }^{3}$ Research Centre on Mathematics \\ and Applications, University of Évora \\ (CIMA-UE), Rua Romão Ramalho, 59, \\ Évora, 7000-671, Portugal \\ Full list of author information is \\ available at the end of the article
}

\begin{abstract}
In this paper, given $f:[a, b] \times(C([a, b]))^{n-2} \times \mathbb{R}^{2} \rightarrow \mathbb{R}$ a $L^{1}$-Carathéodory function, it is considered the functional higher order equation

$$
u^{(n)}(x)=f\left(x, u, u^{\prime}, \ldots, u^{(n-2)}(x), u^{(n-1)}(x)\right)
$$

together with the nonlinear functional boundary conditions, for $i=0, \ldots, n-2$

$$
\begin{aligned}
& L_{i}\left(u, u^{\prime}, \ldots, u^{(n-1)}, u^{(i)}(a)\right)=0, \\
& L_{n-1}\left(u, u^{\prime}, \ldots, u^{(n-1)}, u^{(n-2)}(b)\right)=0 .
\end{aligned}
$$

Here, $L_{i}, i=0, \ldots, n-1$, are continuous functions. It will be proved an existence and location result in presence of not necessarily ordered lower and upper solutions, without assuming any monotone properties on the boundary conditions and on the nonlinearity $f$.
\end{abstract}

\section{Introduction}

In this paper, it is considered the functional higher order boundary value problem, for $n \geq 2$ composed by the equation

$$
u^{(n)}(x)=f\left(x, u, \ldots, u^{(n-3)}, u^{(n-2)}(x), u^{(n-1)}(x)\right)
$$

for a.a. $x \in I:=[a, b]$, where $f: I \times(C(I))^{(n-2)} \times \mathbb{R}^{2} \rightarrow \mathbb{R}$ is a $L^{1}$-Carathéodory function, and the function boundary conditions

$$
\begin{aligned}
& L_{i}\left(u, u^{\prime}, \ldots, u^{(n-1)}, u^{(i)}(a)\right)=0, \quad i=0, \ldots, n-2, \\
& L_{n-1}\left(u, u^{\prime}, \ldots, u^{(n-1)}, u^{(n-2)}(b)\right)=0,
\end{aligned}
$$

where $L_{i}, i=0, \ldots, n-1$, are continuous functions without assuming monotone conditions or another type of variation.

The functional differential equation (1) can be seen as a generalization of several types of full differential and integro-differential equations and allow to consider delays, maxima or minima arguments, or another kind of global variation on the unknown function or its derivatives until order $(n-3)$. On the other hand, the functional dependence in (2) makes possible its application to a huge variety of boundary conditions, such as Lidstone,

\section{照 Springer}

๑) 2013 Fialho and Minhós; licensee Springer. This is an Open Access article distributed under the terms of the Creative Commons Attribution License (http://creativecommons.org/licenses/by/2.0), which permits unrestricted use, distribution, and reproduction in any medium, provided the original work is properly cited. 
separated, multipoint, nonlocal and impulsive conditions, among others. As example, we mention the problems contained in [1-15]. A detailed list about the potentialities of functional problems and some applications can be found in [16].

Recently, functional boundary value problems have been studied by several authors following several approaches, as it can be seen, for example, in [17-24]. In this work, the lower and upper solutions method is applied together with topological degree theory, according some arguments suggested in [25-27].

The novelty of this paper consists in the following items:

- There is no monotone assumptions on the boundary functions $L_{i}, i=0, \ldots, n-1$, by using adequate auxiliary functions and global arguments. This fact with the functional dependence on the unknown function and its derivatives till order $(n-1)$ will allow that problem (1)-(2) can include the periodic and antiperiodic cases, which were not covered by the existent literature on functional boundary value problems. In this sense, the results in this area, as for instance [28-32], are improved, even for $n=2$, where equation (1) loses its functional part.

- No extra condition on the nonlinear part of (1) is considered, besides a Nagumo-type growth assumption. In fact, as far as we know, it is the first time where lower and upper solutions technique is used without such hypothesis on function $f$, by the use of stronger definitions for lower and upper solutions.

- No order between lower and upper solutions is assumed. Putting the 'well ordered' case on adequate auxiliary functions, it allows that lower and upper solutions could be well ordered, by reversed order or without a defined order.

The last section contains an example where the potentialities of the functional dependence on the equation and on the boundary conditions are explored.

\section{Definitions and auxiliary functions}

In this section, it will be introduced the notations and definitions needed forward together with some auxiliary functions useful to construct some ordered functions on the basis of the not necessarily ordered lower and upper solutions of the referred problem.

A Nagumo-type growth condition, assumed on the nonlinear part, will be an important tool to set an a priori bound for the $(n-1)$ th derivative of the corresponding solutions.

In the following, $W^{m, 1}(I)$ denotes the usual Sobolev Spaces in $I$, that is, the subset of $C^{m-1}(I)$ functions, whose $(m-1)$ th derivative is absolutely continuous in $I$ and the $m$ th derivative belongs to $L^{1}(I)$ and the usual norms

$$
\|u\|_{p}= \begin{cases}\left(\int_{0}^{1}|u(x)|^{p} d x\right)^{1 / p}, & 1 \leq p<\infty \\ \sup \{|u(x)|: x \in I\}, & p=\infty\end{cases}
$$

for spaces $L^{p}, 1 \leq p \leq \infty$.

The function $f: I \times(C(I))^{(n-2)} \times \mathbb{R}^{2} \rightarrow \mathbb{R}$ is a $L^{1}$-Carathéodory function, that is, $f(x, \cdot, \ldots, \cdot, \cdot, \cdot)$ is a continuous function for a.e. $x \in I ; f\left(\cdot, y_{0}, \ldots, y_{n-2}, y_{n-1}\right)$ is measurable for $\left(y_{0}, \ldots, y_{n-2}, y_{n-1}\right) \in(C(I))^{(n-2)} \times \mathbb{R}^{2}$; and for every $M>0$ there is a real-valued function $\psi_{M} \in L^{1}(I)$ such that

$$
\left|f\left(x, y_{0}, y_{1}, y_{2}, y_{3}\right)\right| \leq \psi_{M}(x), \quad \text { for a.e. } x \in[0,1]
$$

and for every $\left(y_{0}, y_{1}, y_{2}, y_{3}\right) \in(C(I))^{(n-2)} \times \mathbb{R}^{2}$ with $\left|y_{i}\right| \leq M$, for $i=0, \ldots, n-1$. 
The main tool to obtain the location part is the upper and lower solutions method. However, in this case, they must be defined as a pair, which means that it is not possible to define them independently from each other. Moreover, it is pointed out that lower and upper functions, and the correspondent first derivatives, are not necessarily ordered.

To introduce 'some order', some auxiliary functions must be defined.

For any $\alpha, \beta \in W^{n-2,1}(I)$ define functions $\alpha_{i}, \beta_{i}: I \rightarrow \mathbb{R}, i=0, \ldots, n-3$, as it follows:

$$
\begin{aligned}
& \alpha_{n-3}(x)=\min \left\{\alpha^{(n-3)}(a), \beta^{(n-3)}(a)\right\}+\int_{a}^{x} \alpha^{(n-2)}(s) d s, \\
& \beta_{n-3}(x)=\max \left\{\alpha^{(n-3)}(a), \beta^{(n-3)}(a)\right\}+\int_{a}^{x} \beta^{(n-2)}(s) d s, \\
& \alpha_{i}(x)=\min \left\{\alpha^{(i)}(a), \beta^{(i)}(a)\right\}+\int_{a}^{x} \alpha_{i+1}(s) d s \\
& \beta_{i}(x)=\max \left\{\alpha^{(i)}(a), \beta^{(i)}(a)\right\}+\int_{a}^{x} \beta_{i+1}(s) d s
\end{aligned}
$$

for $i=0, \ldots, n-4$.

The Nagumo-type condition is given by next definition.

Definition 1 Consider $\Gamma_{i}, \gamma_{i} \in C(I), i=0, \ldots, n-2$, such that $\Gamma_{i}(x) \leq \gamma_{i}(x), \forall x \in I$, and the set

$$
E=\left\{\left(x, y_{0}, \ldots, y_{n-1}\right) \in I \times \mathbb{R}^{n}: \gamma_{i}(x) \leq y_{i} \leq \Gamma_{i}(x), i=0, \ldots, n-2\right\} .
$$

A function $f: I \times \mathbb{R}^{n} \rightarrow \mathbb{R}$ is said to verify a Nagumo-type condition in $E$ if there exists $\varphi_{E} \in C([0,+\infty),(0,+\infty))$ such that

$$
\left|f\left(x, y_{0}, \ldots, y_{n-1}\right)\right| \leq \varphi_{E}\left(\left|y_{n-1}\right|\right)
$$

for every $\left(x, y_{0}, \ldots, y_{n-1}\right) \in E$, and

$$
\int_{r}^{+\infty} \frac{t}{\varphi_{E}(t)} d t>\max _{x \in I} \Gamma_{n-2}(x)-\min _{x \in I} \gamma_{n-2}(x),
$$

where $r \geq 0$ is given by

$$
r:=\max \left\{\frac{\Gamma_{n-2}(b)-\gamma_{n-2}(a)}{b-a}, \frac{\Gamma_{n-2}(a)-\gamma_{n-2}(b)}{b-a}\right\} .
$$

The next result gives an a priori estimate for the $(n-1)$ th derivative of all possible solutions of (1).

Lemma 2 There exists $K>0$ such that for every $L^{1}$-Carathéodory function $f: I \times$ $(C(I))^{n-2} \times \mathbb{R}^{2} \rightarrow \mathbb{R}$ satisfying (4) and (5) and every solution $u$ of $(1)$ such that

$$
\gamma_{i}(x) \leq u^{(i)}(x) \leq \Gamma_{i}(x), \quad \text { a.e. } x \in I,
$$


for $i=0, \ldots, n-2$, we have

$$
\left\|u^{(n-1)}\right\|_{\infty}<R
$$

Moreover, the constant $R$ depends only on the functions $\varphi$ and $\gamma_{i}, \Gamma_{i}(i=0, \ldots, n-2)$ and not on the boundary conditions.

Proof The proof is similar to [19, Lemma 2.1].

The upper and lower solution definition is then given by the following.

Definition 3 The functions $\alpha, \beta \in W^{n, 1}(I)$ are a pair of lower and upper solutions for problem (1)-(2) if $\alpha^{(n-2)}(x) \leq \beta^{(n-2)}(x)$, on $I$, for all $\left(v_{0}, \ldots, v_{n-3}\right) \in A:=\left[\alpha_{0}, \beta_{0}\right] \times \cdots \times$ $\left[\alpha_{n-3}, \beta_{n-3}\right]$, and for every $\left(w_{1}, w_{2}\right) \in B:=\left[\alpha^{(n-2)}, \beta^{(n-2)}\right] \times[-K, K]$, for some $K>0$, the following inequalities hold for a.e. $x \in[a, b]$,

$$
\begin{aligned}
& \alpha^{(n)}(x) \geq f\left(x, v_{0}, \ldots, v_{n-3}, \alpha^{(n-2)}(x), \alpha^{(n-1)}(x)\right), \\
& \beta^{(n)}(x) \leq f\left(x, v_{0}, \ldots, v_{n-3}, \beta^{(n-2)}(x), \beta^{(n-1)}(x)\right),
\end{aligned}
$$

and for $j=0, \ldots, n-3$,

$$
\begin{aligned}
& L_{j}\left(v_{0}, \ldots, v_{n-3}, w_{1}, w_{2}, \alpha_{j}(a)\right) \geq 0, \\
& L_{n-2}\left(v_{0}, \ldots, v_{n-3}, w_{1}, w_{2}, \alpha^{(n-2)}(a)\right) \geq 0, \\
& L_{n-1}\left(v_{0}, \ldots, v_{n-3}, w_{1}, w_{2}, \alpha^{(n-2)}(b)\right) \geq 0, \\
& L_{j}\left(v_{0}, \ldots, v_{n-3}, w_{1}, w_{2}, \beta_{j}(a)\right) \leq 0, \\
& L_{n-2}\left(v_{0}, \ldots, v_{n-3}, w_{1}, w_{2}, \beta^{(n-2)}(a)\right) \leq 0, \\
& L_{n-1}\left(v_{0}, \ldots, v_{n-3}, w_{1}, w_{2}, \beta^{(n-2)}(b)\right) \leq 0 .
\end{aligned}
$$

\section{Existence and location result}

In this section, it is provided an existence and location theorem for the problem (1)-(2). More precisely, sufficient conditions are given for, not only the existence of a solution $u$, but also to have information about the location of $u$, and all its derivatives up to the $(n-1)$ order.

The arguments of the proof require the following lemma, given on [29].

Lemma 4 For $v, w \in C(I)$ such that $v(x) \leq w(x)$, for every $x \in I$, define

$$
q(x, u)=\max \{v, \min \{u, w\}\}
$$

Then, for each $u \in C^{1}(I)$ the next two properties hold:

(a) $\frac{d}{d x} q(x, u(x))$ exists for a.e. $x \in I$.

(b) If $u, u_{m} \in C^{1}(I)$ and $u_{m} \rightarrow u$ in $C^{1}(I)$ then

$$
\frac{d}{d x} q\left(x, u_{m}(x)\right) \rightarrow \frac{d}{d x} q(x, u(x)) \text { for a.e. } x \in I \text {. }
$$


Now, we are in a position to prove the main result of this paper.

Theorem 5 Assume that there exists a pair of lower and upper solutions $(\alpha, \beta)$ of problem (1)-(2).

If $: I \times(C(I))^{n-2} \times \mathbb{R}^{2} \rightarrow \mathbb{R}$ is a $L^{1}$-Carathéodory function, satisfying a Nagumo-type condition in

$$
E_{*}=\left\{\begin{array}{l}
\left(x, y_{0}, \ldots, y_{n-1}\right) \in I \times \mathbb{R}^{n-1}: \alpha_{i}(x) \leq y_{i} \leq \beta_{i}(x), \quad i=0, \ldots, n-3, \\
\alpha^{(n-2)}(x) \leq y_{n-2} \leq \beta^{(n-2)}(x),
\end{array}\right.
$$

then problem (1)-(2) has at least one solution $u$ such that

$$
\begin{aligned}
& \alpha_{i}(x) \leq u^{(i)}(x) \leq \beta_{i}(x), \quad i=0, \ldots, n-3, \\
& \alpha^{(n-2)}(x) \leq u^{(n-2)}(x) \leq \beta^{(n-2)}(x), \quad \forall x \in I
\end{aligned}
$$

for every $x \in I$, and $\left|u^{(n-1)}(x)\right| \leq K, \forall x \in I$, where

$$
K=\max \left\{R,\left|\alpha^{(n-1)}(x)\right|,\left|\beta^{(n-1)}(x)\right|\right\}
$$

and $R>0$ is given by $(7)$.

Proof Define the continuous functions, for $i=0, \ldots, n-3$,

$$
\begin{aligned}
& \delta_{i}\left(x, y_{i}\right)=\max \left\{\alpha_{i}(x), \min \left\{y_{i}, \beta_{i}(x)\right\}\right\}, \\
& \delta_{n-2}\left(x, y_{n-2}\right)=\max \left\{\alpha^{(n-2)}(x), \min \left\{y_{n-2}, \beta^{(n-2)}(x)\right\}\right\},
\end{aligned}
$$

and the truncation, not necessarily continuous,

$$
\xi(z)=\max \{-K, \min \{z, K\}\}
$$

with $K$ given by (10).

Consider the modified problem composed by the equation

$$
u^{(n)}(x)=f\left(\begin{array}{c}
x, \delta_{0}(\cdot, u), \ldots, \delta_{n-3}\left(\cdot, u^{(n-3)}\right), \\
\delta_{n-2}\left(x, u^{(n-2)}(x)\right), \xi\left(\frac{d}{d x}\left(\delta_{n-2}\left(x, u^{(n-2)}(x)\right)\right)\right)
\end{array}\right)
$$

and the boundary conditions, for $i=0, \ldots, n-2$,

$$
\begin{aligned}
& u^{(i)}(a)=\delta_{i}\left(a, u^{(i)}(a)+L_{i}\left(\begin{array}{c}
\delta_{0}(\cdot, u), \ldots, \delta_{n-2}\left(\cdot, u^{(n-2)}\right), \\
\xi\left(\frac{d}{d x}\left(\delta_{n-2}\left(\cdot, u^{(n-2)}\right)\right)\right), u^{(i)}(a)
\end{array}\right)\right) \\
& u^{(n-2)}(b)=\delta_{n-2}\left(b, u^{(n-2)}(b)+L_{n-1}\left(\begin{array}{c}
\delta_{0}(\cdot, u), \ldots, \delta_{n-2}\left(\cdot, u^{(n-2)}\right), \\
\xi\left(\frac{d}{d x}\left(\delta_{n-2}\left(\cdot, u^{(n-2)}\right)\right)\right), u^{(n-2)}(b)
\end{array}\right)\right) .
\end{aligned}
$$

The proof will follow the next steps: 
Step 1. Every solution $u$ of problem (12)-(13), satisfies

$$
\begin{aligned}
& \alpha^{(n-2)}(x) \leq u^{(n-2)}(x) \leq \beta^{(n-2)}(x), \\
& \alpha_{i}(x) \leq u^{(i)}(x) \leq \beta_{i}(x), \quad i=0, \ldots, n-3,
\end{aligned}
$$

and $\left|u^{(n-1)}(x)\right|<K$, for every $x \in I$, with $K>0$ given in (10).

Let $u$ be a solution of the modified problem (12)-(13). Assume, by contradiction, that there exists $x \in I$ such that $\alpha^{(n-2)}(x)>u^{(n-2)}(x)$ and let $x_{0} \in I$ be such that

$$
\min _{x \in I}(u-\alpha)^{(n-2)}(x):=(u-\alpha)^{(n-2)}\left(x_{0}\right)<0 .
$$

As, by (13), $u^{(n-2)}(a) \geq \alpha^{(n-2)}(a)$ and $u^{(n-2)}(b) \geq \alpha^{(n-2)}(b)$, then $x_{0} \in(a, b)$. So, there is $\left(x_{1}, x_{2}\right) \subset(a, b)$ such that

$$
\begin{aligned}
& u^{(n-2)}(x)<\alpha^{(n-2)}(x), \quad \forall x \in\left(x_{1}, x_{2}\right), \\
& (u-\alpha)^{(n-2)}\left(x_{1}\right)=(u-\alpha)^{(n-2)}\left(x_{2}\right)=0 .
\end{aligned}
$$

Therefore,

$$
\delta_{n-2}\left(x, u^{(n-2)}(x)\right)=\alpha^{(n-2)}(x), \quad \forall x \in\left(x_{1}, x_{2}\right),
$$

and

$$
\frac{d}{d x} \delta_{n-2}\left(x, u^{(n-2)}(x)\right)=\alpha^{(n-1)}(x), \quad \text { a.e. } x \in\left(x_{1}, x_{2}\right) .
$$

Now, since for all $u \in C^{n-2}(I)$ it is satisfied that $\left(\delta_{0}(\cdot, u), \ldots, \delta_{n-3}\left(\cdot, u^{\prime}\right)\right) \in A$, we deduce that

$$
\begin{aligned}
u^{(n)}(x) & =f\left(\begin{array}{c}
x, \delta_{0}(\cdot, u), \ldots, \delta_{n-3}\left(\cdot, u^{(n-3)}\right), \delta_{n-2}\left(x, u^{(n-2)}(x)\right), \\
\xi\left(\frac{d}{d x}\left(\delta_{n-2}\left(x, u^{(n-2)}(x)\right)\right)\right)
\end{array}\right) \\
& =f\left(x, \delta_{0}(\cdot, u), \ldots, \delta_{n-3}\left(\cdot, u^{(n-3)}\right), \alpha^{(n-2)}(x), \alpha^{(n-1)}(x)\right) \\
& \leq \alpha^{(n)}(x) \quad \text { for a.e. } x \in\left(x_{1}, x_{2}\right) .
\end{aligned}
$$

As $(u-\alpha)^{(n-1)}\left(x_{0}\right)=0$ and $(u-\alpha)^{(n-1)}$ is nonincreasing in $\left(x_{1}, x_{2}\right)$, this contradicts the definitions of $x_{0}$ and $x_{2}$.

The inequality $u^{(n-2)}(x) \leq \beta^{(n-2)}(x)$, in $I$, can be proved in same way and so,

$$
\alpha^{(n-2)}(x) \leq u^{(n-2)}(x) \leq \beta^{(n-2)}(x), \quad \forall x \in I .
$$

By (13) and (3), the following inequalities hold for every $x \in I$ :

$$
\begin{aligned}
u^{(n-3)}(x) & =u^{(n-3)}(a)+\int_{a}^{x} u^{(n-2)}(s) d s \geq \alpha_{n-3}(a)+\int_{a}^{x} \alpha^{(n-2)}(s) d s \\
& \geq \min \left\{\alpha^{(n-3)}(a), \beta^{(n-3)}(a)\right\}+\int_{a}^{x} \alpha^{(n-2)}(s) d s=\alpha_{n-3}(x) .
\end{aligned}
$$

Analogously, it can be obtained $u^{(n-3)}(x) \leq \beta_{n-3}(x)$, for $x \in I$. 
The remaining inequalities are obtained by the same integration process. Applying previous bounds in Lemma 2, and remarking that

$$
\int_{r}^{K} \frac{s}{\varphi(s)} d s \geq \int_{r}^{R} \frac{s}{\varphi(s)} d s
$$

for $K$ given by (10), it is obtained, by Lemma 2, the a priori bound $\left|u^{(n-1)}(x)\right|<K$, for $x \in I$. For details, see [33, Lemma 2].

Step 2. Problem (12)-(13) has at least one solution.

For $\lambda \in[0,1]$ let us consider the homotopic problem given by

$$
u^{(n)}(x)=\lambda f\left(\begin{array}{c}
x, \delta_{0}(\cdot, u), \ldots, \delta_{n-3}\left(\cdot, u^{(n-3)}\right), \\
\delta_{n-2}\left(x, u^{(n-2)}(x)\right), \xi\left(\frac{d}{d x}\left(\delta_{n-2}\left(x, u^{(n-2)}(x)\right)\right)\right)
\end{array}\right)
$$

and the boundary conditions, for $i=0, \ldots, n-2$,

$$
\begin{aligned}
u^{(i)}(a)= & \lambda \delta_{i}\left(a, u^{(i)}(a)+L_{i}\left(\begin{array}{c}
\delta_{0}(\cdot, u), \ldots, \delta_{n-2}\left(\cdot, u^{(n-2)}\right), \\
\xi\left(\frac{d}{d x}\left(\delta_{n-2}\left(\cdot, u^{(n-2)}\right)\right)\right), u^{(i)}(a)
\end{array}\right)\right):=\lambda L_{A_{i}}, \\
u^{(n-2)}(b) & =\lambda \delta_{n-2}\left(b, u^{(n-2)}(b)+L_{n-1}\left(\begin{array}{c}
\delta_{0}(\cdot, u), \ldots, \delta_{n-2}\left(\cdot, u^{(n-2)}\right), \\
\xi\left(\frac{d}{d x}\left(\delta_{n-2}\left(\cdot, u^{(n-2)}\right)\right)\right), u^{(n-2)}(b)
\end{array}\right)\right) \\
: & =\lambda L_{B} .
\end{aligned}
$$

Let us consider the norms in $C^{n-1}(I)$ and in $L^{1}(I) \times \mathbb{R}^{n}$, respectively,

$$
\|v\|_{C^{n-1}}=\max \left\{\|v\|_{\infty}, \ldots,\left\|v^{(n-1)}\right\|_{\infty}\right\}
$$

and

$$
\left|\left(h, h_{1}, \ldots, h_{n}\right)\right|=\max \left\{\|h\|_{L^{1}}, \max \left\{\left|h_{1}\right|, \ldots,\left|h_{n}\right|\right\}\right\} .
$$

Define the operators $\mathcal{L}: W^{n, 1}(I) \subset C^{n-1}(I) \rightarrow L^{1}(I) \times \mathbb{R}^{n}$ by $\mathcal{L} u=\left(u^{(n)}, u(a), \ldots, u^{(n-2)}(a)\right.$, $\left.u^{(n-2)}(b)\right)$ and, for $\lambda \in[0,1], i=0, \ldots, n-2, \mathcal{N}_{\lambda}: C^{n-1}(I) \rightarrow L^{1}(I) \times \mathbb{R}^{n}$ by

$$
\mathcal{N}_{\lambda} u=\left(\lambda f\left(\begin{array}{c}
x, \delta_{0}(\cdot, u), \ldots, \delta_{n-3}\left(\cdot, u^{(n-3)}\right), \delta_{n-2}\left(x, u^{(n-2)}(x)\right), \\
\xi\left(\frac{d}{d x}\left(\delta_{n-2}\left(x, u^{(n-2)}(x)\right)\right)\right) \\
\lambda L_{A_{1}}, \ldots, \lambda L_{A_{n-2}}, \lambda L_{B}
\end{array}\right),\right.
$$

Since $L_{0}, \ldots, L_{n-1}$ are continuous and $f$ is a $L^{1}$-Carathéodory function, then, from Lemma 4 , $\mathcal{N}_{\lambda}$ is continuous. Moreover, as $\mathcal{L}^{-1}$ is compact, it can be defined the completely continuous operator $\mathcal{T}_{\lambda}: C^{n-1}(I) \rightarrow C^{n-1}(I)$ by $\mathcal{T}_{\lambda} u=\mathcal{L}^{-1} \mathcal{N}_{\lambda}(u)$.

It is obvious that the fixed points of operator $\mathcal{T}_{\lambda}$ coincide with the solutions of problem (16)-(17).

As $\mathcal{N}_{\lambda} u$ is bounded in $L^{1}(I) \times \mathbb{R}^{n}$ and uniformly bounded in $C^{n-1}(I)$, we have that any solution of the problem (16)-(17), verifies the following a priori bound

$$
\|u\|_{C^{n-1}} \leq\left\|\mathcal{L}^{-1}\right\|_{C^{n-1}}\left|\mathcal{N}_{\lambda}(u)\right| \leq \bar{K},
$$

for some $\bar{K}>0$ independent of $\lambda$. 
In the set $\Omega=\left\{u \in C^{n-1}(I):\|u\|_{C^{n-1}}<\bar{K}+1\right\}$, the degree $d\left(\mathcal{I}-\mathcal{T}_{\lambda}, \Omega, 0\right)$ is well defined for every $\lambda \in[0,1]$ and, by the invariance under homotopy, $d\left(\mathcal{I}-\mathcal{T}_{0}, \Omega, 0\right)=d\left(\mathcal{I}-\mathcal{T}_{1}, \Omega, 0\right)$. As the equation $x=\mathcal{T}_{0}(x)$ is equivalent to the problem

$$
\left\{\begin{array}{l}
u^{(n)}(x)=0, \\
u^{(i)}(a)=u^{(n-2)}(b)=0, \quad i=0, \ldots, n-2,
\end{array}\right.
$$

which has only the trivial solution, then $d\left(\mathcal{I}-\mathcal{T}_{0}, \Omega, 0\right)= \pm 1$. So, by degree theory, the equation $x=\mathcal{T}_{1}(x)$ has at least one solution, that is, the problem (12)-(13) has at least a solution in $\Omega$.

Step 3. Every solution $u$ of problem (12)-(13) is a solution of (1)-(2).

Let $u$ be a solution of the modified problem (12)-(13). By previous steps, function $u$ fulfills equation (1). So, it will be enough to prove the following inequalities, for $i=0, \ldots, n-3$ :

$$
\begin{aligned}
& \alpha_{i}(a) \leq u^{(i)}(a)+L_{i}\left(\begin{array}{c}
\delta_{0}(\cdot, u), \ldots, \delta_{n-2}\left(x, u^{(n-2)}(x)\right), \\
\xi\left(\frac{d}{d x}\left(\delta_{n-2}\left(x, u^{(n-2)}(x)\right)\right)\right), u^{(i)}(a)
\end{array}\right) \leq \beta_{i}(a), \\
& \alpha^{(n-2)}(a) \leq u^{(n-2)}(a)+L_{n-1}\left(\begin{array}{c}
\delta_{0}(\cdot, u), \ldots, \delta_{n-2}\left(x, u^{(n-2)}(x)\right), \\
\xi\left(\frac{d}{d x}\left(\delta_{n-2}\left(x, u^{(n-2)}(x)\right)\right)\right), u^{(n-2)}(a)
\end{array}\right) \\
& \leq \beta^{(n-2)}(a)
\end{aligned}
$$

and

$$
\begin{aligned}
\alpha^{(n-2)}(b) & \leq u^{(n-2)}(b)+L_{n-1}\left(\begin{array}{c}
\delta_{0}(\cdot, u), \ldots, \delta_{n-2}\left(x, u^{(n-2)}(x)\right), \\
\xi\left(\frac{d}{d x}\left(\delta_{n-2}\left(x, u^{(n-2)}(x)\right)\right)\right), u^{(n-2)}(b)
\end{array}\right) \\
& \leq \beta^{(n-2)}(b) .
\end{aligned}
$$

Assume that

$$
u(a)+L_{0}\left(\begin{array}{l}
\delta_{0}(\cdot, u), \ldots, \delta_{n-2}\left(x, u^{(n-2)}(x)\right), \\
\xi\left(\frac{d}{d x}\left(\delta_{n-2}\left(x, u^{(n-2)}(x)\right)\right)\right), u(a)
\end{array}\right)>\beta_{0}(a) .
$$

Then, by (13), $u(a)=\beta_{0}(a)$. By previous steps, it is obtained the following contradiction with (18):

$$
\begin{aligned}
& u(a)+L_{0}\left(\begin{array}{c}
\delta_{0}(\cdot, u), \ldots, \delta_{n-2}\left(x, u^{(n-2)}(x)\right), \\
\xi\left(\frac{d}{d x}\left(\delta_{n-2}\left(x, u^{(n-2)}(x)\right)\right)\right), u(a)
\end{array}\right) \\
& =\beta_{0}(a)+L_{0}\left(\begin{array}{c}
\delta_{0}(\cdot, u), \ldots, \delta_{n-2}\left(x, u^{(n-2)}(x)\right), \\
\xi\left(\frac{d}{d x}\left(\delta_{n-2}\left(x, u^{(n-2)}(x)\right)\right)\right), \beta_{0}(a)
\end{array}\right) \\
& \leq \beta_{0}(a) .
\end{aligned}
$$

Applying similar arguments, it can be proved that

$$
\alpha_{0}(a) \leq u(a)+L_{0}\left(\begin{array}{l}
\delta_{0}(\cdot, u), \ldots, \delta_{n-2}\left(x, u^{(n-2)}(x)\right), \\
\xi\left(\frac{d}{d x}\left(\delta_{n-2}\left(x, u^{(n-2)}(x)\right)\right)\right), u(a)
\end{array}\right)
$$


and analogously, for $j=1, \ldots, n-3$,

$$
\alpha_{j}(a) \leq u^{(j)}(a)+L_{j}\left(\begin{array}{c}
\delta_{0}(\cdot, u), \ldots, \delta_{n-2}\left(x \cdot, u^{(n-2)}(x)\right), \\
\xi\left(\frac{d}{d x}\left(\delta_{n-2}\left(x, u^{(n-2)}(x)\right)\right)\right), u^{(j)}(a)
\end{array}\right) \leq \beta_{j}(a) .
$$

Also, using the same arguments and the same techniques, it can be proved that

$$
\begin{aligned}
\alpha^{(n-2)}(a) & \leq u^{(n-2)}(a)+L_{n-1}\left(\begin{array}{c}
\delta_{0}(\cdot, u), \ldots, \delta_{n-2}\left(x, u^{(n-2)}(x)\right), \\
\xi\left(\frac{d}{d x}\left(\delta_{n-2}\left(x, u^{(n-2)}(x)\right)\right)\right), u^{(n-2)}(a)
\end{array}\right) \\
& \leq \beta^{(n-2)}(a), \\
\alpha^{(n-2)}(b) & \leq u^{(n-2)}(b)+L_{n-1}\left(\begin{array}{c}
\delta_{0}(\cdot, u), \ldots, \delta_{n-2}\left(x, u^{(n-2)}(x)\right), \\
\xi\left(\frac{d}{d x}\left(\delta_{n-2}\left(x, u^{(n-2)}(x)\right)\right)\right), u^{(n-2)}(b)
\end{array}\right) \\
& \leq \beta^{(n-2)}(b) .
\end{aligned}
$$

\section{Example}

This section contains a problem composed by an integro-differential equation with some functional boundary conditions, whose solvability is proved in presence of nonordered lower and upper solutions. We remark that such fact was not possible with the results in the current literature. This example does not model any particular problem arising in real phenomena. Our purpose consists on emphasizing the powerful of the developed theory in this paper by showing what kind of problems we can deal with.

Consider, for $x \in[0,1]$, the fourth-order equation

$$
u^{(i v)}(x)=\int_{0}^{x} u(s) d s+\max _{x \in[0,1]}\left\{u^{\prime}(x)\right\}+\left(u^{\prime \prime}(x)\right)^{3}-\left(u^{\prime \prime \prime}(x)+1\right)^{\frac{2}{3}}
$$

coupled with the boundary value conditions

$$
\begin{aligned}
& -\min _{x \in[0,1]} u^{\prime \prime}(x)-26 u(0)=0, \\
& u(s)-(u(0))^{3}+14=0, \\
& \max _{x \in[0,1]} u(x)-2 u^{\prime \prime}(0)=0, \\
& \sqrt[3]{u^{\prime \prime}(1)}=0
\end{aligned}
$$

One can verify that functions

$$
\alpha(x)=-\frac{x^{3}}{6}-12 x^{2}+20 x-1 \quad \text { and } \quad \beta(x)=\frac{x}{3}^{3}+12 x^{2}+1
$$

are, respectively, lower and upper solutions for the problem (19)-(20). Moreover, we deduce that

$$
\begin{array}{ll}
\alpha_{1}(x)=-\frac{x}{2}^{2}-24 x-\frac{9}{2}, & \alpha_{0}(x)=-\frac{x^{3}}{6}-12 x^{2}-\frac{9}{2} x-1, \\
\beta_{1}(x)=x^{2}+24 x+25, & \beta_{0}(x)=\frac{x^{3}}{3}+12 x^{2}+125 x+\frac{40}{3}
\end{array}
$$


and

$$
\begin{aligned}
& f\left(x, y_{0}, y_{1}, y_{2}, y_{3}\right)=\int_{0}^{x} y_{0}(s) d s+\max _{x \in[0,1]}\left\{y_{1}(x)\right\}+\left(y_{2}(x)\right)^{3}-\left(y_{3}(x)+1\right)^{\frac{2}{3}} \\
& L_{0}\left(z_{1}, z_{2}, z_{3}, z_{4}, z_{5}\right)=-\min _{x \in[0,1]} z_{3}-26 z_{5} \\
& L_{1}\left(z_{1}, z_{2}, z_{3}, z_{4}, z_{5}\right)=z_{1}-\left(z_{5}\right)^{3}+14 \\
& L_{2}\left(z_{1}, z_{2}, z_{3}, z_{4}, z_{5}\right)=\max _{x \in[0,1]} z_{1}-2 z_{5} \\
& L_{3}\left(z_{1}, z_{2}, z_{3}, z_{4}, z_{5}\right)=-\sqrt[3]{z_{5}}
\end{aligned}
$$

As the continuous function $f$ verifies (4) and (5) for $\varphi_{E_{*}}\left(y_{3}\right)=\frac{1,847}{12}+\left(y_{3}+1\right)^{\frac{2}{3}}$ in

$$
E_{*}=\left\{\begin{array}{c}
\left(x, y_{0}, y_{1}, y_{2}, y_{3}\right) \in[0,1] \times \mathbb{R}^{4}: \\
-\frac{x}{6}^{3}-12 x^{2}-\frac{9}{2} x-1 \leq y_{0} \leq \frac{x}{3}^{3}+12 x^{2}+125 x+\frac{40}{3} \\
-\frac{x}{2}^{2}-24 x-\frac{9}{2} \leq y_{1} \leq x^{2}+24 x+25 \\
-x-24 \leq y_{2} \leq 2 x+24
\end{array}\right\}
$$

then, by Theorem 5 , there is a nontrivial solution $u$ for problem (19)-(20) such that

$$
\begin{aligned}
& -\frac{x^{3}}{6}-12 x^{2}-\frac{9}{2} x-1 \leq u(x) \leq \frac{x^{3}}{3}+12 x^{2}+125 x+\frac{40}{3} \\
& -\frac{x^{2}}{2}-24 x-\frac{9}{2} \leq u^{\prime}(x) \leq x^{2}+24 x+25 \\
& -x-24 \leq u^{\prime \prime}(x) \leq 2 x+24
\end{aligned}
$$

for all $x \in[0,1]$.

\section{Competing interests}

The authors declare that they have no competing interests.

\section{Authors' contributions}

The work presented here was carried out in collaboration between the authors. The authors contributed to every part of this study equally, read and approved the final version of the manuscript.

\section{Author details}

'School of Mathematics, Physics and Technology, College of the Bahamas, Nassau, Bahamas. ${ }^{2}$ Department of Mathematics, School of Sciences and Technology, University of Évora, Évora, Portugal. ${ }^{3}$ Research Centre on Mathematics and Applications, University of Évora (CIMA-UE), Rua Romão Ramalho, 59, Évora, 7000-671, Portugal.

\section{Acknowledgements}

Dedicated to Professor Jean Mawhin on the occasion of his 70th anniversary.

Received: 15 December 2012 Accepted: 22 March 2013 Published: 10 April 2013

\section{References}

1. Cabada, A, Grossinho, MR, Minhós, F: On the solvability of some discontinuous third order nonlinear differential equations with two point boundary conditions. J. Math. Anal. Appl. 285, 174-190 (2003)

2. Cuia, Y, Sun, J: Existence of multiple positive solutions for fourth-order boundary value problems in Banach spaces. Bound. Value Probl. 2012, Article ID 107 (2012)

3. Feng, H, Ji, D, Ge, W: Existence and uniqueness of solutions for a fourth-order boundary value problem. Nonlinear Anal. 70, 3561-3566 (2009)

4. Franco, D, O’Regan, D, Perán, J: Fourth-order problems with nonlinear boundary conditions. J. Comput. Appl. Math. $174,315-327(2005)$

5. Graef, JR, Kong, L, Yang, B: Existence of solutions for a higher order multi-point boundary value problems. Results Math. 53, 77-101 (2009)

6. Han, J, Liu, Y, Zhao, J: Integral boundary value problems for first order nonlinear impulsive functional integro-differential differential equations. Appl. Math. Comput. 218, 5002-5009 (2012) 
7. Kong, L, Wong, J: Positive solutions for higher order multi-point boundary value problems with nonhomogeneous boundary conditions. J. Math. Anal. Appl. 367, 588-611 (2010)

8. $L u, H$, Sun, L, Sun, J: Existence of positive solutions to a non-positive elastic beam equation with both ends fixed. Bound. Value Probl. 2012, Article ID 56 (2012)

9. $\mathrm{Ma}, \mathrm{D}$, Yang, X: Upper and lower solution method for fourth-order four-point boundary value problems. J. Comput. Appl. Math. 223, 543-551 (2009)

10. Minhós, F, Gyulov, T, Santos, Al: Lower and upper solutions for a fully nonlinear beam equations. Nonlinear Anal. 71 , 281-292 (2009)

11. Pang, $\mathrm{H}, \mathrm{Ge}, \mathrm{W}$ : Existence results for some fourth order multi-point boundary value problem. Math. Comput. Model. 49, 1319-1325 (2009)

12. Pao, CV, Wang, YM: Fourth-order boundary value problems with multi-point boundary conditions. Commun. Appl. Nonlinear Anal. 16, 1-22 (2009)

13. Pei, $M$, Chang, S, Oh, YS: Solvability of right focal boundary value problems with superlinear growth conditions. Bound. Value Probl. 2012, Article ID 60 (2012)

14. Zhang, X, Liu, L: Positive solutions of fourth-order multi-point boundary value problems with bending term. Appl. Math. Comput. 194, 321-332 (2007)

15. Zhao, $\mathrm{H}$ : A note on upper and lower solutions method for fourth-order boundary value problems. Ann. Differ. Equ. $24,117-120$ (2008)

16. Cabada, A, Pouso, R, Minhós, F: Extremal solutions to fourth-order functional boundary value problems including multipoint condition. Nonlinear Anal., Real World Appl. 10, 2157-2170 (2009)

17. Cabada, A, Fialho, J, Minhós, F: Non ordered lower and upper solutions to fourth order functional BVP. Discrete Contin. Dyn. Syst. 2011, suppl. 209-218 (2011) (Issue Special)

18. Cabada, A, Minhós, F: Fully nonlinear fourth order equations with functional boundary conditions. J. Math. Anal. Appl. 340(1), 239-251 (2008)

19. Cabada, A, Minhós, F, Santos, Al: Solvability for a third order discontinuous fully equation with functional boundary conditions. J. Math. Anal. Appl. 322, 735-748 (2006)

20. Graef, J, Kong, L, Minhós, F: Higher order functional boundary value problems: existence and location results. Acta Sci. Math. 77, 87-100 (2011)

21. Graef, J, Kong, L, Minhós, F: Higher order boundary value problems with $\phi$-Laplacian and functional boundary conditions. Comput. Math. Appl. 61, 236-249 (2011)

22. Sun, Y, Han, Z: On forced oscillation of higher order functional differential equations. Appl. Math. Comput. $\mathbf{2 1 8}$ 6966-6971 (2012)

23. Wang, W, Shen, J, Luo, Z: Multi-point boundary value problems for second-order functional differential equations. Comput. Math. Appl. 56, 2065-2072 (2008)

24. Zhao, Z, Liang, J: Existence of solutions to functional boundary value problem of second-order nonlinear differential equation. J. Math. Anal. Appl. 373, 614-634 (2011)

25. Minhós, F, Fialho, J: On the solvability of some fourth-order equations with functional boundary conditions. Discrete Contin. Dyn. Syst. 2009, suppl. 564-573 (2009)

26. Graef, J, Kong, L, Minhós, F, Fialho, J: On lower and upper solutions method for higher order functional boundary value problems. Appl. Anal. Discrete Math. 5(1), 133-146 (2011)

27. Mawhin, J: Topological Degree Methods in Nonlinear Boundary Value Problems. Regional Conference Series in Mathematics, vol. 40. Am. Math. Soc., Providence (1979)

28. Minhós, F: Periodic solutions for some fully nonlinear fourth order differential equations. Discrete Contin. Dyn. Syst. 2011, suppl. 1068 (2011) (Issue Special)

29. Wang, MX, Cabada, A, Nieto, JJ: Monotone method for nonlinear second order periodic boundary value problems with Carathéodory functions. Ann. Pol. Math. 58, 221-235 (1993)

30. Weng, S, Gao, H, Jiang, D, Hou, X: Upper and lower solutions method for fourth-order periodic boundary value problems. J. Appl. Anal. 14, 53-61 (2008)

31. Yao, M, Zhao, A, Yan, J: Anti-periodic boundary value problems of second order impulsive differential equations. Comput. Math. Appl. 59, 3617-3629 (2010)

32. Zhang, Y: The existence of solutions to nonlinear second order periodic boundary value problems. Nonlinear Anal. 76, 140-152 (2013)

33. Grossinho, MR, Minhós, F, Santos, Al: A note on a class of problems for a higher order fully nonlinear equation under one sided Nagumo type condition. Nonlinear Anal. 70, 4027-4038 (2009) 